\title{
Invasion of the canalis sinuosus by dental implants: A report of 3 cases
}

\author{
Werner Harumiti Shintaku(D) ${ }^{1, *}$, Cimara Fortes Ferreira $\mathbb{D}^{2}$, Jaqueline de Souza Venturin $\mathbb{D}^{1}$ \\ ${ }^{1}$ Department of Diagnostic Sciences, University of Tennessee Health Sciences College of Dentistry, Memphis, TN, USA \\ ${ }^{2}$ Department of Periodontology, University of Tennessee Health Sciences College of Dentistry, Memphis, TN, USA
}

\begin{abstract}
The canalis sinuosus (CS) and its accessory canals (ACs) are anatomical structures in the anterior maxilla. These structures are often neglected when planning implant surgery because their clinical significance is still not well-defined. After a retrospective evaluation of 194 patients rehabilitated with dental implants in the anterior maxilla, 3 patients were identified who presented unexpected chronic neurosensory disturbances without any clinical signs supportive of implant failure. Tomographic assessment using cone-beam computed tomography (CBCT) revealed the invasion of the CS and ACs by dental implants, which appeared to explain the patients' symptoms. The purpose of this report was to familiarize practicing dentists and specialists with the CS and its ACs. Unanticipated neurosensory symptoms after implant placement in the anterior maxilla justify the use of CBCT to rule out an injury to this neurovascular bundle.
\end{abstract}

(Imaging Sci Dent 2020; 50: 353-7)

KEY WORDS: Cone-Beam Computed Tomography; Dental Implants; Maxillary Nerve; Pain

Awareness of the anatomical structures before any surgical procedure is essential for safe and satisfactory outcomes of dental treatments. ${ }^{1}$ The placement of dental implants should follow a thorough evaluation of the area of interest, in which the configuration of these structures is assessed and possible anatomical variations are identified. In dentistry, the analysis of anatomical bony structures and bone quality is promptly obtainable with the 3-dimensional (3D) images generated by cone-beam computed tomography (CBCT).

Recently, an anatomical structure has gained attention due to the increased frequency of placement of dental implants in the anterior maxilla. This structure was termed the canalis sinuosus (CS), due to its convoluted path, by Jones in $1939 .^{2}$ The CS originates from the infraorbital foramen and runs laterally beside the nasal cavity. ${ }^{3,4} \mathrm{It}$ contains the anterior superior alveolar nerve and vessels that innervate and supply nutrition to the maxillary incisors, canines, and adjacent tissues. ${ }^{2-8}$ The presence of ac-

Received May 25, 2020; Revised June 28, 2020; Accepted July 21, 2020 *Correspondence to : Dr. Werner Harumiti Shintaku

Department of Diagnostic Sciences, University of Tennessee Health Science Center, College of Dentistry, 875 Union Avenue, Memphis, TN 38163, USA

Tel) 1-901-448-2845,E-mail)wshintak@uthsc.edu cessory canals (ACs), known as palatine extensions, at the end of the CS has been reported. ${ }^{9}$ These ACs, usually 1-2 $\mathrm{mm}$ in diameter, connect the CS to the alveolar ridge of the anterior hard palate at the level of the maxillary incisors and canines with variable anatomy. ${ }^{9-12}$

Preoperative conventional 2-dimensional radiographs cannot always be used to identify the CS and ACs. ${ }^{13,14}$ The CS and ACs are visible on CBCT scans, but their clinical relevance is still questionable due to a lack of information in the current literature. Even once identified, it is still unclear whether the invasion of the CS and ACs, for example by a dental implant, can cause neurosensory disorders. Therefore, the purpose of this report was to familiarize practicing dentists and specialists with the CS and its ACs by presenting 3 cases in which patients reported unexpected postoperative symptoms after implant placement in the anterior maxilla.

\section{Case Report}

\section{Case 1}

A CBCT scan of a 73-year-old Caucasian man reporting postoperative discomfort (dysesthesia) in the right 


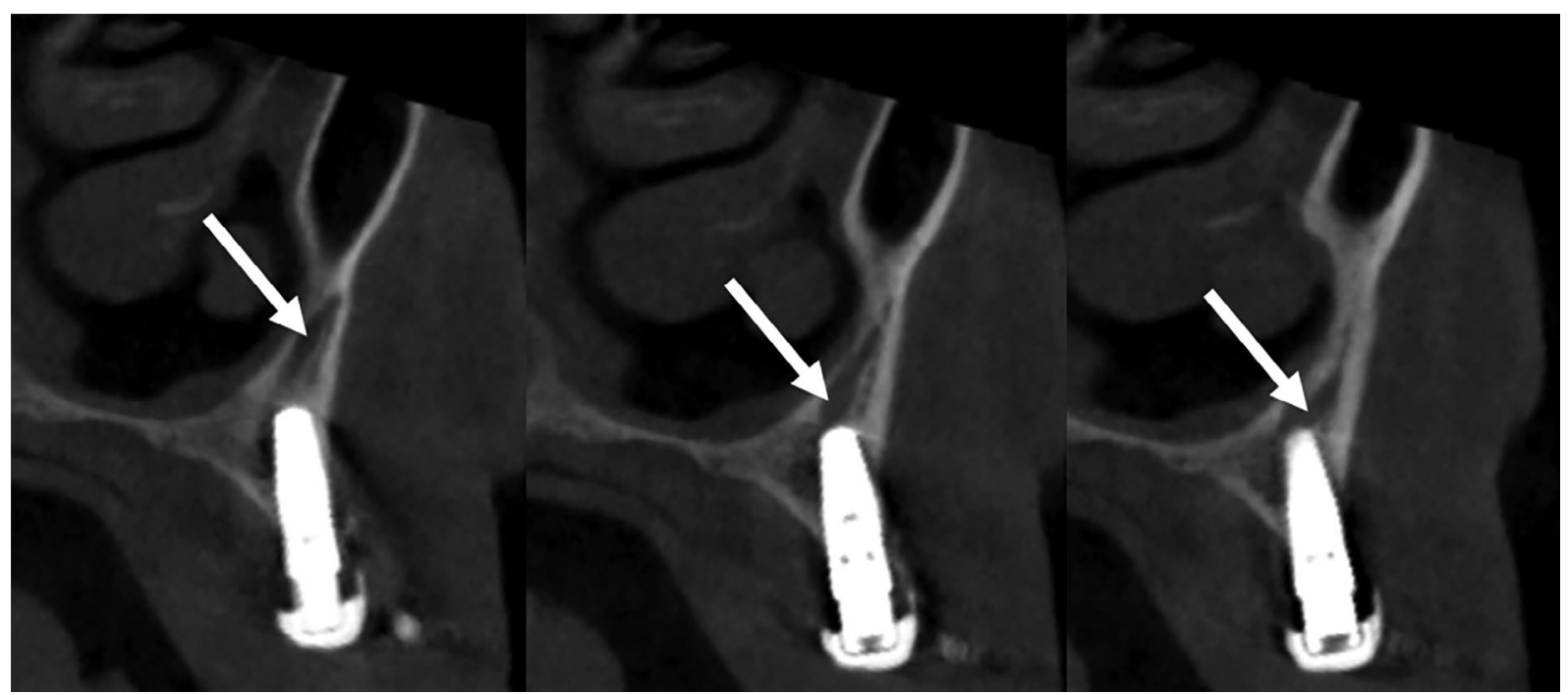

Fig. 1. Cross-sectional images of the right anterior maxilla. An implant in the canine region interrupts the right canalis sinuosus.

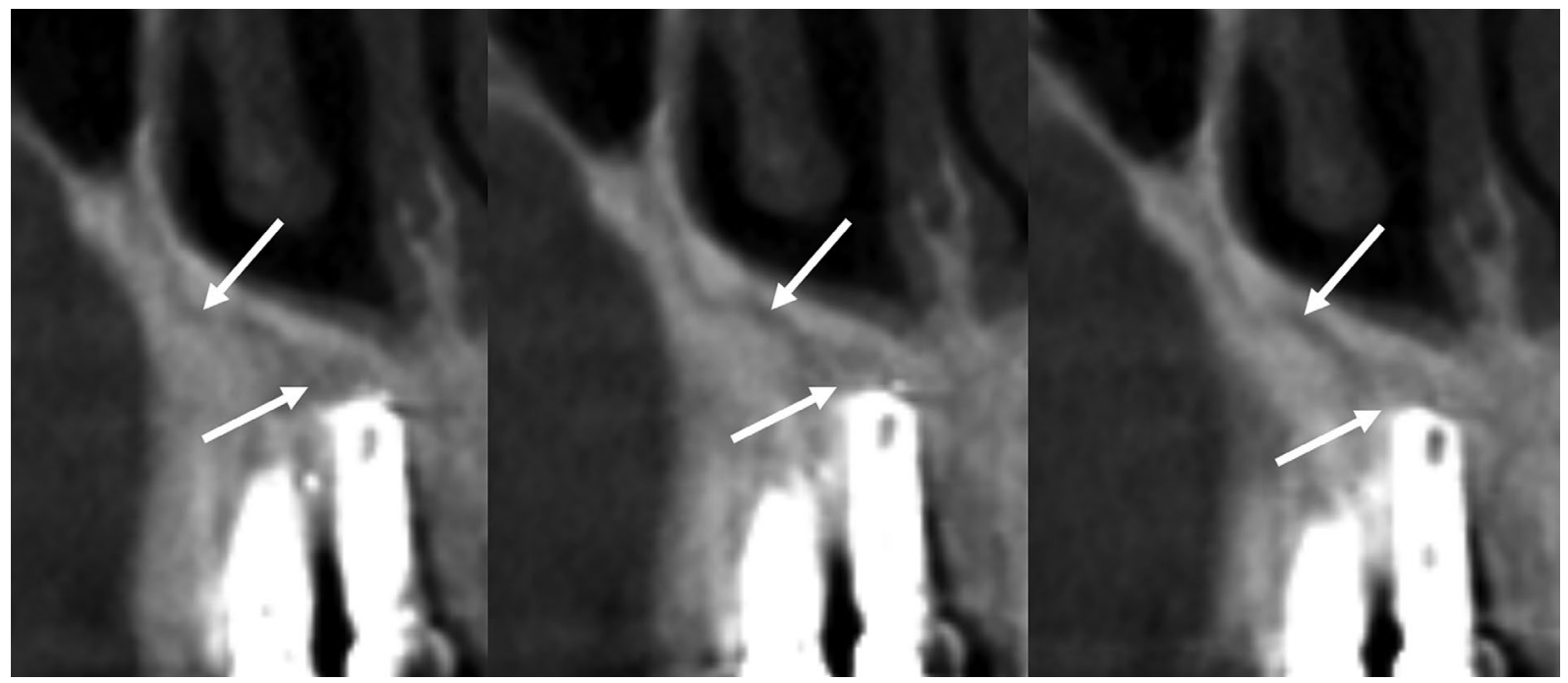

Fig. 2. Coronal images of the right anterior maxilla. An implant in the central incisor region interrupts the right canalis sinuosus.

maxillary canine region was acquired. A medium-volume CBCT exam $\left(10 \mathrm{~cm} \times 10 \mathrm{~cm}, 0.3 \mathrm{~mm}^{3}\right.$ voxel size $)$ of the anterior maxilla had been previously obtained using a CS 9300 machine (Carestream, Atlanta, GA, USA) at 90 $\mathrm{kVp}, 4 \mathrm{~mA}$, and an 8-second exposure time. The volume was stored in MiPacs (Medicor Imaging, Charlotte, NC, USA), a picture archiving and communication system (PACS), using Digital Imaging and Communication in Medicine (DICOM) standards. The images were evaluated by a board-certified oral and maxillofacial radiologist, a board-certified periodontist, and an orofacial pain specialist by consensus using OnDemand 3D software (Cybermed Inc., Seoul, Korea) in a dimly lit room on a 27-inch Dell U2715 monitor (Dell Inc., Round Rock, TX, USA).

The images were analyzed in coronal, axial, sagittal, and cross-sectional reconstructions and revealed an implant replacing the right maxillary canine. The apex of the implant interrupted the CS (Fig. 1). The remaining implant surfaces were well-integrated, and the peri-implant bone was radiographically unremarkable. No signs of fractures of the buccal or palatal plates were present. The gingival tissues appeared healthy. Other clinical and radiographic findings supportive of reported symptoms were not present. A diagnosis of invasion of the CS by the implant as the cause for the patient's discomfort was 


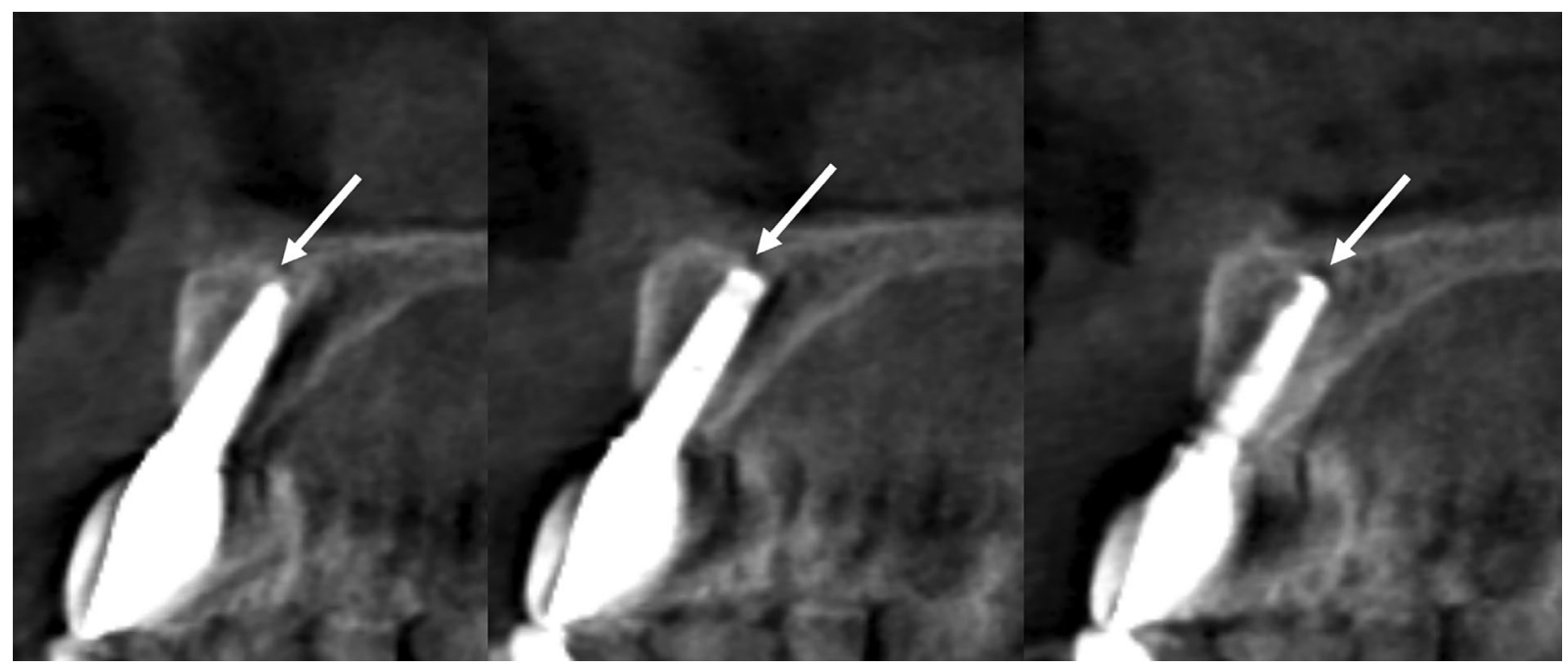

Fig. 3. Cross-sectional images of the right anterior maxilla. An implant in the lateral incisor region interrupts the right canalis sinuosus.

reached based on the clinical signs and symptoms and the radiographic findings.

\section{Case 2}

A CBCT scan of a 47-year-old Caucasian woman reporting occasional pain and a feeling of "tightness in the bone" in the right anterior maxilla after implant placement was acquired. The patient did not report that local trauma had occurred, and the rest of her medical history was noncontributory. A medium-volume CBCT exam of the anterior maxilla was acquired, stored, and assessed as in the previous case.

The imaging data were analyzed in coronal, axial, sagittal, and cross-sectional views and showed an implant replacing the right central incisor. The implant interrupted the right CS apically (Fig. 2). Despite the presence of imaging artifacts, the remaining implant surfaces showed apposition with the alveolar bone support. Clinically, the implant was stable. The buccal or palatal cortical plates were intact. An implant was also observed to be replacing the right lateral incisor and was radiographically unremarkable. The gingival tissues appeared healthy. Other clinical and radiographic findings supportive of the reported symptoms were not present. A diagnosis of invasion of the CS by the implant as the cause for the patient's complaint was determined based on the clinical signs and symptoms and the radiographic findings.

\section{Case 3}

A CBCT scan of a 78-year-old Caucasian man reporting a sharp pain upon chewing associated with an implant replacing the right lateral incisor was acquired. The patient's medical status was otherwise unremarkable, and the patient denied that any local trauma had occurred. A medium-volume CBCT exam of the anterior maxilla was obtained, stored, and assessed using the same protocol as in cases 1 and 2.

The volumetric images were analyzed in coronal, axial, sagittal, and cross-sectional reconstructions and revealed an interruption of the CS at the apical portion of the implant (Fig. 3). The remaining implant surfaces were well-osseointegrated. The peri-implant bone was radiographically unremarkable. Signs of fractures of the buccal or palatal plates were absent. Other clinical and radiographic findings supportive of the reported symptoms were absent. A diagnosis of invasion of the CS by the implant as the cause for the patient's complaint was reached based on the clinical signs and symptoms and the radiographic findings.

\section{Discussion}

Imaging is a non-invasive diagnostic tool that is essential for planning implant treatment. Some anatomic structures and their variations are only visible on more advanced imaging exams, and their presence can directly influence treatment outcomes. As supported by the current literature, 3D images, especially high-resolution CBCT images, provide reliable visualization of bony structures and are recommended by the American Academy of Oral and Maxillofacial Radiology as the standard radiographic projections for preoperative dental implant treatment 
planning. ${ }^{15}$

Before any surgical procedure, it is essential to identify variations in the anatomical structures and their locations to avoid complications and undesired symptoms. ${ }^{1,11}$ An invasion of major neurovascular canals may potentially result in significant bleeding and sensory disorders, such as temporary or permanent paresthesia, hyperesthesia, dysesthesia, altered sensation, and persistent chronic pain. ${ }^{1,16,17}$ Specifically for dental implants, the contact of an implant with neural tissue may compromise the osseointegration of the implant and, most importantly, cause neurosensory disorders. ${ }^{18,19}$ Pain is the most common symptom of neurosensory disorders and is usually temporary after an injury. ${ }^{16,20}$ However, unexpected long-lasting neuropathies may occur in some cases. ${ }^{21,22}$

Implant-related injuries of major neurovascular canals, such as the nasopalatine canal, the incisive mandibular canal, and the mandibular canal, are well documented in the literature. ${ }^{5,16}$ Reports of injuries of smaller neurovascular bundles, such as the CS and its ACs, are still rare because the identification of these smaller canals may be challenging and is often neglected in dental surgical procedures. $^{21,22}$ Articles reporting unexpected bleeding after injuries to relatively small vessels are available, but these articles do not mention neurosensory complications. ${ }^{16,18,23}$

The CS has a close relationship with the anterior superior alveolar nerve. ${ }^{1-3,5}$ ACs connect the CS to the palatal aspect of the alveolar ridge of the anterior hard palate, typically at the level of the central and lateral incisors and canines. ${ }^{1,6}$ These canals provide innervation and nutrition to the anterior teeth and soft tissues. ${ }^{9}$ Unfortunately, specific information regarding injuries to the CS and its ACs is still minimal and inconclusive.

As stated in a retrospective study, the prevalence of implant-related perforations of adjacent anatomical structures was significantly higher in the maxilla than in the mandible. ${ }^{24}$ Despite those findings, the significance of these perforations could not be determined due to the lack of clinical information. In the present retrospective study, 3 patients reporting unexpected neurosensory symptoms after implant placement in the anterior maxilla were identified. Our study complements the publication of Gaêta-Araujo ${ }^{24}$ by demonstrating the clinical implication of injuries to the CS and its ACs, as supported by clinical and radiographic findings.

Finally, these cases demonstrate the vital role of radiographic exams in the determination of the likely cause of a patient's long-lasting symptoms, and this report proposes that the CS and its ACs are noteworthy anatomic struc- tures. If unexpected neurosensory symptoms occur after implant placement in the anterior maxilla, CBCT is valuable for ruling out an injury to this neurovascular bundle and for providing information to facilitate corrective procedures.

\section{Conflicts of Interest: None}

\section{References}

1. Gurler G, Delilbasi C, Ogut EE, Aydin K, Sakul U. Evaluation of the morphology of the canalis sinuosus using cone-beam computed tomography in patients with maxillary impacted canines. Imaging Sci Dent 2017; 47: 69-74.

2. Jones FW. The anterior superior alveolar nerve and vessels. J Anat 1939; 73: 583-91.

3. Shelley AM, Rushton VE, Horner K. Canalis sinuosus mimicking a periapical inflammatory lesion. Br Dent J 1999; 186: 378-9.

4. Manhães Júnior LR, Villaça-Carvalho MF, Moraes ME, Lopes SL, Silva MB, Junqueira JL. Location and classification of canalis sinuosus for cone beam computed tomography: avoiding misdiagnosis. Braz Oral Res 2016; 30: e49.

5. Neves FS, Crusoé-Souza M, Franco LC, Caria PH, Bonfim-Almeida P, Crusoé-Rebello I. Canalis sinuosus: a rare anatomical variation. Surg Radiol Anat 2012; 34: 563-6.

6. de Oliveira-Santos C, Rubira-Bullen IR, Monteiro SA, León JE, Jacobs R. Neurovascular anatomical variations in the anterior palate observed on CBCT images. Clin Oral Implants Res 2013; 24: 1044-8.

7. Torres MG, de Faro Valverde L, Vidal MT, Crusoe-Rebello IM. Branch of the canalis sinuosus: a rare anatomical variation - a case report. Surg Radiol Anat 2015; 37: 879-81.

8. Arruda JA, Silva P, Silva L, Álvares P, Silva L, Zavanelli R, et al. Dental implant in the canalis sinuosus: a case report and review of the literature. Case Rep Dent 2017; 2017: 4810123.

9. Ferlin R, Pagin BS, Yaedú RY. Canalis sinuosus: a systematic review of the literature. Oral Surg Oral Med Oral Pathol Oral Radiol 2019; 127: 545-51.

10. Hu KS, Kwak HH, Song WC, Kang HJ, Kim HC, Fontaine C, et al. Branching patterns of the infraorbital nerve and topography within the infraorbital space. J Craniofac Surg 2006; 17 : 1111-5.

11. Machado VC, Chrcanovic BR, Felippe MB, Manhães Júnior LR, de Carvalho PS. Assessment of accessory canals of the canalis sinuosus: a study of 1000 cone beam computed tomography examinations. Int J Oral Maxillofac Surg 2016; 45: 1586-91.

12. von Arx T, Lozanoff S, Sendi P, Bornstein MM. Assessment of bone channels other than the nasopalatine canal in the anterior maxilla using limited cone beam computed tomography. Surg Radiol Anat 2013; 35: 783-90.

13. Kohavi D. Demostration of unusually wide artery in the maxillary alveolar bone using a reformatting program of computed tomography: a case report. Int J Oral Maxillofac Implants 1994; 9: 444-8. 
14. Temmerman A, Hertelé S, Teughels W, Dekeyser C, Jacobs R, Quirynen M. Are panoramic images reliable in planning sinus augmentation procedures? Clin Oral Implants Res 2011; 22: 189-94.

15. Tyndall DA, Price JB, Tetradis S, Ganz SD, Hildebolt C, Scarfe WC, et al. Position statement of the American Academy of Oral and Maxillofacial Radiology on selection criteria for the use of radiology in dental implantology with emphasis on cone beam computed tomography. Oral Surg Oral Med Oral Pathol Oral Radiol 2012; 113: 817-26.

16. Kalpidis CD, Setayesh RM. Hemorrhaging associated with endosseous implant placement in the anterior mandible: a review of the literature. J Periodontol 2004; 75: 631-45.

17. Rodella LF, Buffoli B, Labanca M, Rezzani R. A review of the mandibular and maxillary nerve supplies and their clinical relevance. Arch Oral Biol 2012; 57: 323-34.

18. Liang X, Jacobs R, Lambrichts I. An assessment on spiral CT scan of the superior and inferior genial spinal foramina and canals. Surg Radiol Anat 2006; 28: 98-104.

19. Bornstein MM, Balsiger R, Sendi P, von Arx T. Morphology of the nasopalatine canal and dental implant surgery: a radiographic analysis of 100 consecutive patients using limited cone-beam computed tomography. Clin Oral Implants Res 2011; 22: 295301.
20. Al-Sabbagh M, Okeson JP, Khalaf MW, Bhavsar I. Persistent pain and neurosensory disturbance after dental implant surgery: pathophysiology, etiology, and diagnosis. Dent Clin North Am 2015; 59: 131-42.

21. De Rouck T, Collys K, Cosyn J. Single-tooth replacement in the anterior maxilla by means of immediate implantation and provisionalization: a review. Int J Oral Maxillofac Implants 2008; 23: 897-904.

22. Gunaseelan R, Anantanarayanan P, Veerabahu M, Vikraman $\mathrm{B}$, Sripal R. Intraoperative and perioperative complications in anterior maxillary osteotomy: a retrospective evaluation of 103 patients. J Oral Maxillofac Surg 2009; 67: 1269-73.

23. Tepper G, Hofschneider UB, Gahleitner A, Ulm C. Computed tomographic diagnosis and localization of bone canals in the mandibular interforaminal region for prevention of bleeding complications during implant surgery. Int J Oral Maxillofac Implants 2001; 16: 68-72.

24. Gaêta-Araujo H, Oliveira-Santos N, Mancini AX, Oliveira ML, Oliveira-Santos C. Retrospective assessment of dental implant-related perforations of relevant anatomical structures and inadequate spacing between implants/teeth using conebeam computed tomography. Clin Oral Investig 2020; 24 : 3281-8. 